\title{
Developing Data Quality Aware Applications
}

\author{
Ricardo Pérez, César Guerra-García, Mario Piattini and Ma Ángeles Moraga \\ Institute of Information Technologies \& Systems \\ University of Castilla-La Mancha \\ Ciudad Real, Spain \\ \{ricardo.pdelcastillo, mario.piattini, mariaangeles.moraga\}@uclm.es \\ cesararturo.guerra@alu.uclm.es
}

\begin{abstract}
Inadequate levels of Data Quality (DQ) in Information Systems (IS) suppose a very important problem for organizations. In any case, they look for to assure data quality from earlier stages on information system developments. This paper proposes to incorporate mechanisms into software development methodologies, in order to integrate users DQ requirements aimed at assuring the data quality from the beginning of development. It brings a framework consisting of processes, activities and tasks, well defined, which would be incorporated in existent software development methodology, as METRICA V3; and therefore, to assure software product data quality created according to this methodology. The extension presented, is a guideline, and this can be extended and applied to other development methodologies like Unified Development Process.
\end{abstract}

Keywords: Software Quality Assurance, Software Development Methodologies, Data Quality.

\section{INTRODUCTION}

Organizations must assure the quality of data that uses, because it is the raw material of their business processes [1]. Researches such as [2], [3], [4], [5, 6], shows that poor Data Quality is a serious problem in many organizations. Furthermore, [5] and [7] registers quantitative information: The cost of data quality problems is $8-25 \%$ of an organization's revenue. $40-50 \%$ of the ICT budget of company is dedicated to solving problems associated with a low quality of the data handling. $11 \%$ is the percentage of firms in U.S. that have programs for managing data quality and $48 \%$ still not have plans for managing the DQ.

In both academic and industrial environments, there is a growing interest in Data Quality (DQ) because it has been proven to be a great asset for organizations. Moreover, poor data and information quality will have a negative impact on the global efficiency of organization [8],[9], because it can affect in multiple way to the organizational performance.

If organizations want to mitigate the impacts of poor data quality, they must assure the quality of data in their information systems, because the data are their inputs and outputs. Moreover, it would be desirable to advance data quality assurance, from the data definition, to the process of building software products. In other words, to add processes, techniques and tools at software development methodologies to assure, at certain levels, the data quality in information systems developed, according to this methodology. "Organizations do not need to wait for problems to appear before they address them. If they know what to look for, organizations can anticipate and handle $I Q$ problems before they trigger a crisis" [10]. One reason for doing this is that prevention DQ costs are lower than detection or repair DQ cost [11].

For our purposes, this data quality assurance can be understood from the point of view of the requirements for software to be developed. The data quality assurance has an important benefit: By introducing data quality management issues since earlier phases of the IS life cycle, a better performance regarding data quality can be obtained for the operational behavior of IS.

This paper presents an interface to support the data quality management for a specific development methodology as METRICA V3 [12]. METRICA V3 was developed by the Spanish Public Administration (analogous to the methodologies French MERISE [13] or English SSADM [14]. This interface consists of auxiliary processes and activities that support other main processes in software development methodology. These processes assure that the Information Systems development take into account aspects of data quality. In the second section, presents an introduction of methodology METRICA V3. In third section is presented the interface proposal to assure data quality. Finally, are exposed the conclusions of this work.

\section{OVERVIEW OF METRICA V3}

As noted, METRICA V3 [12] is a methodology for software project development, which also covers maintenance. METRICA V3 allows handle aspects and topics to ensure that a project accomplishes according to the planned objectives in terms of quality and cost. Moreover, the methodology covers both structured as object oriented developments.

METRICA V3 is based on the ISO 12207 "Information technology - software life cycle processes" [15], which inherits its structure-oriented processes, activities and tasks. It also takes into account other international software development standards, such as ISO / IEC TR 15.504/SPICE "Software Process Improvement and Assurance Standards Capability Determination" [16], 
UNE-EN-ISO 9001:2000 “Systems Quality Management Requirements" [17], UNE-EN-ISO 9000:2000 "Systems Quality Management - Fundamentals and Vocabulary" [18]. METRICA V3 consists of several processes, as well as a set of interfaces for implementation to other organizational support processes. The following paragraphs describe each processes and interfaces.

\section{A. Main Processes}

METRICA V3 is composed of three main processes: Information System Planning (ISP), Information System Development (ISD) and Information System Maintenance (ISM). In addition, ISD process can be broken down into other processes [12].

- $\quad$ [ISP] Information System Planning. This process orients actions in the field of Information System Development, of which the main objective is supporting corporate strategy. At the end, the output of this process will be a project plan with the specific scope of the project to be developed.

- [ISD] Information System Development. The aim of this process is to develop the information system itself. This process is the central process and it is formed up by the following subprocesses.

- [SVS] System Viability Study. The purpose of this process is to examine a specific set of user requirements in order to propose a tactical solution taking into account economical, technical, legal and operational criteria to evaluate the best choice among all of possible solutions: develop a new Information System or adapt one of commercial software package according to customer requirements.

- [ISA] Information System Analysis. The objective of this process is to get IS detailed specification, by means of a catalogue of requirements and a set of models to meet the user information requirements to those who will develop the information system and will be the entry to Information System Design process.

- [ISD] Information System Design. The goal is to get the definition of system architecture and technological environment that will give support, together with the detailed components specification. From here on, generate all construction specifications relating to system itself.

- [ISC] Information System Construction. It aims to achieve the construction and testing various components of the information system, starting with the set of logical and physical specifications. - [SIA] System Implantation and Acceptance. The main objective in this case is delivery and acceptance of the system as a whole. Moreover, in this process, is carried out activities necessary for the transition to production system.

- $\quad[$ ISM $]$ Information System Maintenance. The aim of this process is to obtain a new Information System version, based on the needs of maintenance. These requirements can arise from system problems detected or by the need to improve itself.

\section{B. Interfaces (Organizational and Support Processes)}

METRICA V3 includes several interfaces, which define a set auxiliary processes and activities with welldefined objectives, to improve and influence to execution of the main processes in the methodology. These interfaces will guarantee project developed successfully with this methodology. METRICA V3 defines the following four interfaces [12]:

- $\quad[\mathrm{PMI}]$ Project Management Interface. This includes activities which are aimed at planning, monitoring, execution and control of the project plans and revision and evaluation and will be executed parallel to the main ones of the development lifecycle.

- $\quad$ [SI] Security Interface. It incorporates security mechanisms at information systems in addition to those proposed in the methodology itself.

- [QAI] Quality Assurance Interface. It describes the activities required to evaluate external quality of the developed products. The intrinsic quality is to be guaranteed by the main processes of METRICA V3. This interface is closely related to the Data Quality Interface presented in this paper.

- [CMI] Configuration Management Interface. It consists in the application of administrative and technical procedures to IS development and maintenance. The objective is to identify, define and provide information, and monitoring changes in the system configuration, as well as modifications and system versions throughout the lifecycle.

\section{A DATA QUALITY INTERFACE (DQI) FOR METRICA V3}

The objective of the Data Quality Interface is to establish a framework that allows to assurance data quality in a developing project. The interface is designed to get a Data Quality Assurance Plan (DQAP). The DQAP will ensure the quality of data in the software product to be built according to METRICA V3, through introduction user requirements planned to assure data quality. The DQAP consists of a set of activities planned in parallel with the system development, aimed at achieving two objectives [9]: (1) To prevent, reduce and eliminate data quality deficiencies in the data used by the software product obtained in operation. (2) To achieve reasonable confidence that the benefits and services expected by the customer, or user, are satisfied, and if they fail, it is not by data uses deficiencies. The interface is structured in the following processes and activities. In the next sections, in each activity is provided a table which it specifies the following information: for each activity, is indicated the tasks which it is composed. For each task, is specified the deliverables that are produced, as well as the participants 
involved. In addition, specifying the possible techniques or tools of the methodology that can be used to achieve the deliverables.

\section{A. Study of Viability of Applying a Data Quality Plan}

[SVS-DQ] Study of Viability for Applying a Data Quality Plan. The objective of this process is to determine the viability of applying this Data Quality Interface to the developing project. This process is break down into three activities.

[SVS-DQ 1] Preparation of Data Quality Environment. The purpose of this activity is to enumerate the elements participating in data quality. Thus, it defines the data quality environment (see Table I).

TABLE I. SVS-DQ 1 TASKS

\begin{tabular}{|c|c|c|c|}
\hline Tasks & Artefact & $\begin{array}{c}\text { Techniques } \\
\text { and } \\
\text { practices }\end{array}$ & Stakeholder \\
\hline $\begin{array}{ll}\text { Constitution } & \text { of the } \\
\text { Data } & \text { Quality } \\
\text { Assurance } & \text { Team } \\
\text { (DQAT) } & \end{array}$ & $\begin{array}{l}\text {-Data Quality } \\
\text { Assurance } \\
\text { Team } \\
\text {-DQ Plan }\end{array}$ & -Planning & $\begin{array}{l}\text {-Project } \\
\text { Manager }\end{array}$ \\
\hline $\begin{array}{l}\text { Establishing roles } \\
\text { and designation of } \\
\text { responsibilities }\end{array}$ & $\begin{array}{l}\text { Data } \\
\text { Quality Plan }\end{array}$ & $\begin{array}{l}\text {-Working } \\
\text { sessions } \\
\text { - Planning }\end{array}$ & $\begin{array}{l}\text {-DQAT } \\
\text {-Project } \\
\text { Manager }\end{array}$ \\
\hline $\begin{array}{l}\text { Establishing desired data } \\
\text { quality properties for } \\
\text { data in the User } \\
\text { Requirement } \\
\text { Specifications }\end{array}$ & $\begin{array}{l}\text { Data } \\
\text { Quality Plan }\end{array}$ & $\begin{array}{l}\text {-Working } \\
\text { sessions } \\
\text { - Planning }\end{array}$ & $\begin{array}{l}\text {-DQAT } \\
\text {-Project } \\
\text { Manager }\end{array}$ \\
\hline $\begin{array}{l}\text { Identification of } \\
\text { Information System } \\
\text { components which } \\
\text { are object of the data } \\
\text { quality study }\end{array}$ & $\begin{array}{l}\text {-Scope of } \\
\text { data quality }\end{array}$ & $\begin{array}{l}\text {-Working } \\
\text { sessions } \\
\text { - Planning }\end{array}$ & $\begin{array}{l}\text {-DQAT } \\
\text {-Project } \\
\text { Manager }\end{array}$ \\
\hline $\begin{array}{l}\text { Election of data } \\
\text { quality standards }\end{array}$ & $\begin{array}{l}\text {-Data quality } \\
\text { standards } \\
\text { register }\end{array}$ & $\begin{array}{l}\text {-Working } \\
\text { sessions } \\
\text { - Planning }\end{array}$ & $\begin{array}{l}\text {-DQAT } \\
\text {-Project } \\
\text { Manager }\end{array}$ \\
\hline
\end{tabular}

[SVS-DQ 2] Determining of the present state of data quality. In order to determine if corrective actions are needed, it is necessary to compare the present state of data quality with what is desired or intended [19]. So, the objective of this group of activities is to determine the present state of data quality by studying different ambits of the system (see Table II).

TABLE II. SVS-DQ 2 TASKS

\begin{tabular}{|c|c|c|c|}
\hline Tasks & Artefact & $\begin{array}{c}\text { Techniques } \\
\text { and practices }\end{array}$ & Stakeholder \\
\hline $\begin{array}{l}\text { Assessment of } \\
\text { customer satisfaction } \\
\text { with the present state } \\
\text { of data quality. }\end{array}$ & $\begin{array}{l}\text {-Data } \\
\text { Quality } \\
\text { state review }\end{array}$ & $\begin{array}{l}\text {-Interview } \\
\text {-Estimation } \\
\text {-Meeting }\end{array}$ & $\begin{array}{l}\text {-Customer } \\
\text {-DQAT } \\
\text {-Project } \\
\text { Manager }\end{array}$ \\
\hline $\begin{array}{l}\text { Assessment of state } \\
\text { of data quality } \\
\text { definition }\end{array}$ & $\begin{array}{l}\text {-Data } \\
\text { Quality } \\
\text { state review }\end{array}$ & $\begin{array}{l}\text {-Working } \\
\text { sessions } \\
\text {-Estimation }\end{array}$ & $\begin{array}{l}\text {-DQAT } \\
\text {-Project } \\
\text { Manager }\end{array}$ \\
\hline $\begin{array}{l}\text { Assessment of state } \\
\text { of intrinsic data } \\
\text { quality }\end{array}$ & $\begin{array}{l}\text {-Data } \\
\text { Quality } \\
\text { state review }\end{array}$ & $\begin{array}{l}\text {-Working } \\
\text { sessions } \\
\text {-Estimation }\end{array}$ & $\begin{array}{l}\text {-DQAT } \\
\text {-Project } \\
\text { Manager }\end{array}$ \\
\hline $\begin{array}{l}\text { Assessment of state } \\
\text { of data working } \\
\text { processes. }\end{array}$ & $\begin{array}{l}\text {-Data } \\
\text { Quality } \\
\text { state review }\end{array}$ & $\begin{array}{l}\text {-Working } \\
\text { sessions } \\
\text {-Estimation }\end{array}$ & $\begin{array}{l}\text {-DQAT } \\
\text {-Project } \\
\text { Manager }\end{array}$ \\
\hline
\end{tabular}

[SVS-DQ 3] Determine viability of the implantation of the Data Quality Assurance Plan (DQAP). The objective of this process is to compare estimated costs in terms of resource to run the DQAP or other solutions with estimated costs of non data quality (see Table III).

TABLE III. SVS-DQ 3 TASKS

\begin{tabular}{|l|l|l|l|}
\hline \multicolumn{1}{|c|}{ Tasks } & \multicolumn{1}{|c|}{ Artefact } & $\begin{array}{l}\text { Techniques } \\
\text { and } \\
\text { practices }\end{array}$ & Stakeholder \\
\hline $\begin{array}{l}\text { Estimation of non } \\
\text { data quality costs }\end{array}$ & $\begin{array}{l}\text {-Budget } \\
\text { review }\end{array}$ & -Estimation & $\begin{array}{l}\text {-Project } \\
\text { Manager }\end{array}$ \\
\hline $\begin{array}{l}\text { Estimation of } \\
\text { DQAP costs }\end{array}$ & $\begin{array}{l}\text {-Budget } \\
\text { review }\end{array}$ & -Estimation & $\begin{array}{l}\text {-DQAT } \\
\text {-Project } \\
\text { Manager }\end{array}$ \\
\hline $\begin{array}{l}\text { Estimation of cost } \\
\text { of each alternative } \\
\text { solutions to the } \\
\text { DQAP proposed }\end{array}$ & $\begin{array}{l}\text {-Budget } \\
\text { review }\end{array}$ & -Estimation & $\begin{array}{l}\text {-DQAT } \\
\text {-Project } \\
\text { Manager }\end{array}$ \\
\hline $\begin{array}{l}\text { Determining the } \\
\text { viability of the } \\
\text { DQAP }\end{array}$ & $\begin{array}{l}\text {-Budget } \\
\text { review } \\
\text {-DQ review }\end{array}$ & $\begin{array}{l}\text {-Estimation } \\
\text { Analysis }\end{array}$ & $\begin{array}{l}\text {-DQAT } \\
\text {-Project } \\
\text { Manager }\end{array}$ \\
\hline
\end{tabular}

\section{B. Information System Analysis - Data Quality}

[ISA-DQ] Information System Analysis - Data Quality. Once the necessity to define a DQAP has been accepted and its viability has been decided upon, its content must be specified in detail. The objectives of this process is to review, correct and complete the user requirements catalogue and information models with other news related to data quality or to verify data quality dimensions extracted from a revision of user requirements and data quality standards. All of the next Analysis DQAP activities must be planned throughout the analysis development process.

[ISA-DQ 1] Initial Specification of the DQAP. The DQAP consists of a review of user requirements in order to complete the user requirements catalogue with data quality aspects. The following activities propose an order for these reviews and completions of the requirements:

[ISA-DQ 1.1] Review of the Specification of Data Quality Requirements. The review of the specification comprises several activities, all of them made from data quality perspective (see Table IV).

[ISA-DQ 1.2] Completion of the Requirements for the Data Quality Assurance. Having determined the state of user requirements for data quality it is necessary to complete these specifications with other requirements that could be necessary or that arise from the data quality standards and were not included in the catalogue of requirements. The completion of the specification comprises several activities, all of them made from data quality perspective (see Table V).

[ISA-DQ 2] Review and Extension of Data Model. Early new data quality requirements must be mapped into developing system data components through next corresponding activities, in order to assurance data quality in data (see Table VI). 
TABLE IV. ISA-DQ 1.1 TASKS

\begin{tabular}{|c|c|c|c|}
\hline Tasks & Artefact & $\begin{array}{c}\text { Techniques } \\
\text { and practices }\end{array}$ & Stakeholder \\
\hline $\begin{array}{l}\text { To review of } \\
\text { data } \\
\text { obtaining } \\
\text { procedures }\end{array}$ & $\begin{array}{l}\text {-Review of data } \\
\text { obtaining } \\
\text { procedures plan }\end{array}$ & $\begin{array}{l}\text {-Working } \\
\text { sessions } \\
\text {-Cataloguing }\end{array}$ & $\begin{array}{l}\text {-DQAT } \\
\text {-Project } \\
\text { Manager } \\
\text {-Project } \\
\text { Team }\end{array}$ \\
\hline $\begin{array}{l}\text { To review of } \\
\text { data storage } \\
\text { procedures }\end{array}$ & $\begin{array}{l}\text {-Review of data } \\
\text { obtaining storage } \\
\text { plan }\end{array}$ & $\begin{array}{l}\text {-Working } \\
\text { sessions } \\
\text {-Cataloguing }\end{array}$ & $\begin{array}{l}\text {-DQAT } \\
\text {-Project } \\
\text { Manager } \\
\text {-Project } \\
\text { Team }\end{array}$ \\
\hline $\begin{array}{l}\text { To review of } \\
\text { data } \\
\text { operating } \\
\text { procedures }\end{array}$ & $\begin{array}{l}\text {-Review of data } \\
\text { operating } \\
\text { procedures plan }\end{array}$ & $\begin{array}{l}\text {-Working } \\
\text { sessions } \\
\text {-Cataloguing }\end{array}$ & $\begin{array}{l}\text {-DQAT } \\
\text {-Project } \\
\text { Manager } \\
\text {-Project } \\
\text { Team }\end{array}$ \\
\hline $\begin{array}{l}\text { To review of } \\
\text { requirements } \\
\text { for user } \\
\text { interfaces }\end{array}$ & $\begin{array}{l}\text {-Scope of data } \\
\text { quality }\end{array}$ & $\begin{array}{l}\text {-Working } \\
\text { sessions } \\
\text {-Cataloguing }\end{array}$ & $\begin{array}{l}\text {-User } \\
\text {-DQAT } \\
\text {-Project } \\
\text { Manager } \\
\end{array}$ \\
\hline $\begin{array}{l}\text { Review of } \\
\text { requirements } \\
\text { for hardware } \\
\text { and software } \\
\text { interfaces }\end{array}$ & $\begin{array}{l}\text {-Data quality } \\
\text { standards register }\end{array}$ & $\begin{array}{l}\text {-Working } \\
\text { sessions }\end{array}$ & $\begin{array}{l}\text {-DQAT } \\
\text {-Project } \\
\text { Manager } \\
\text {-Project } \\
\text { Team }\end{array}$ \\
\hline
\end{tabular}

TABLE V. ISA-DQ 1.2 TASKS

\begin{tabular}{|l|l|l|l|}
\hline \multicolumn{1}{|c|}{ Tasks } & Artefact & $\begin{array}{l}\text { Techniques } \\
\text { and } \\
\text { practices }\end{array}$ & Stakeholder \\
\hline $\begin{array}{l}\text { Determining the data } \\
\text { quality dimensions }\end{array}$ & $\begin{array}{l}\text {-DQAP } \\
\text { review }\end{array}$ & $\begin{array}{l}\text {-Working } \\
\text { sessions } \\
\text {-Formal } \\
\text { review }\end{array}$ & $\begin{array}{l}\text {-DQAT } \\
\text {-Project } \\
\text { Manager }\end{array}$ \\
\hline $\begin{array}{l}\text { Completion of data } \\
\text { obtaining procedures } \\
\text { requirements }\end{array}$ & $\begin{array}{l}\text {-DQAP } \\
\text { review }\end{array}$ & $\begin{array}{l}\text {-Working } \\
\text { sessions } \\
\text {-Cataloguing }\end{array}$ & $\begin{array}{l}\text {-DQAT } \\
\text {-Project Manager } \\
\text {-Project Team }\end{array}$ \\
\hline $\begin{array}{l}\text { Completion of data } \\
\text { storage requirements }\end{array}$ & $\begin{array}{l}\text {-DQAP } \\
\text { review }\end{array}$ & $\begin{array}{l}\text {-Working } \\
\text { sessions }\end{array}$ & $\begin{array}{l}\text {-DQAT } \\
\text {-Project } \\
\text { Manager } \\
\text {-Project Team }\end{array}$ \\
\hline $\begin{array}{l}\text { Completion of data } \\
\text { operating } \\
\text { requirements }\end{array}$ & $\begin{array}{l}\text {-DQAP } \\
\text { review }\end{array}$ & $\begin{array}{l}\text {-Working } \\
\text { sessions } \\
\text {-Cataloguing }\end{array}$ & $\begin{array}{l}\text {-DQAT } \\
\text {-Project Manager } \\
\text {-Project Team }\end{array}$ \\
\hline $\begin{array}{l}\text { Completion of } \\
\text { requirements for user } \\
\text { interfaces }\end{array}$ & $\begin{array}{l}\text {-DQAP } \\
\text { review }\end{array}$ & $\begin{array}{l}\text {-Working } \\
\text { sessions } \\
\text {-Cataloguing }\end{array}$ & $\begin{array}{l}\text {-User } \\
\text {-DQAT } \\
\text {-Project Manager }\end{array}$ \\
\hline $\begin{array}{l}\text { Completion of DQ } \\
\text { requirements for } \\
\text { hardware } \\
\text { software interfaces }\end{array}$ & -DQAP \\
review & $\begin{array}{l}\text {-Working } \\
\text { sessions }\end{array}$ & $\begin{array}{l}\text {-DQAT } \\
\text {-Project Manager } \\
\text {-Project Team }\end{array}$ \\
\hline
\end{tabular}

TABLE VI. ISA-DQ 2 TASKS

\begin{tabular}{|c|c|c|c|}
\hline Tasks & Artefact & $\begin{array}{c}\text { Techniques and } \\
\text { practices }\end{array}$ & Stakeholder \\
\hline $\begin{array}{l}\text { Extension of the } \\
\text { Entity/Relationship } \\
\text { Model with data } \\
\text { quality characteristics }\end{array}$ & $\begin{array}{l}-\mathrm{E} / \mathrm{R} \text { model } \\
\text { extension. } \\
\text {-Data Model } \\
\text { review }\end{array}$ & $\begin{array}{l}\text {-Working } \\
\text { sessions } \\
\text {-Formal } \\
\text { review }\end{array}$ & $\begin{array}{l}\text {-DQAT } \\
\text {-Project } \\
\text { Team }\end{array}$ \\
\hline $\begin{array}{ll}\text { Extension } & \text { of the } \\
\text { internal } & \text { semantic } \\
\text { restrictions. } & \\
\end{array}$ & $\begin{array}{l}\text {-Data Model } \\
\text { review }\end{array}$ & $\begin{array}{l}\text {-Working } \\
\text { sessions } \\
\text {-Formal review }\end{array}$ & $\begin{array}{l}\text {-DQAT } \\
\text {-Project } \\
\text { Team }\end{array}$ \\
\hline $\begin{array}{l}\text { Review and } \\
\text { Extension of initial } \\
\text { system data load. }\end{array}$ & $\begin{array}{l}\text {-Data Model } \\
\text { review }\end{array}$ & $\begin{array}{l}\text {-Working } \\
\text { sessions } \\
\text {-Technical } \\
\text { review }\end{array}$ & $\begin{array}{l}\text {-DQAT } \\
\text {-Project } \\
\text { Team }\end{array}$ \\
\hline
\end{tabular}

[ISA-DQ 3] Review and Extension of Process Model. As before, data quality requirements must be into developing system model process components through these activities, reviewing and completing it (see Table VII).

TABLE VII. ISA-DQ 3 TASKS

\begin{tabular}{|c|c|c|c|}
\hline Tasks & Artefact & $\begin{array}{c}\text { Techniques } \\
\text { and } \\
\text { practices }\end{array}$ & Stakeholder \\
\hline $\begin{array}{l}\text { Review and } \\
\text { Extension of the } \\
\text { Process Model with } \\
\text { External Semantic } \\
\text { restrictions. }\end{array}$ & $\begin{array}{l}\text {-Process } \\
\text { Model } \\
\text { review }\end{array}$ & $\begin{array}{l}\text {-Working } \\
\text { sessions } \\
\text {-Formal } \\
\text { review }\end{array}$ & $\begin{array}{l}\text {-DQAT } \\
\text {-Project } \\
\text { Team }\end{array}$ \\
\hline
\end{tabular}

[ISA-DQ 4] Review and Extension of Interfaces. As result of tasks ISA-DQ 1.2.5 and ISA-DQ 1.2.6, data quality requirements catalogue for interfaces was completed. The objective of this process is to translate these requirements into specifications so that interfaces are not a non data quality origin (see Table VIII).

TABLE VIII. ISA-DQ 4 TASKS

\begin{tabular}{|l|l|l|l|}
\hline \multicolumn{1}{|c|}{ Tasks } & Artefact & $\begin{array}{l}\text { Techniques } \\
\text { and } \\
\text { practices }\end{array}$ & Stakeholder \\
\hline $\begin{array}{l}\text { Review and Extension } \\
\text { of User Interfaces }\end{array}$ & $\begin{array}{l}\text {-DQAP } \\
\text { review }\end{array}$ & $\begin{array}{l}\text {-Meeting } \\
\text {-Working } \\
\text { sessions }\end{array}$ & $\begin{array}{l}\text {-User } \\
\text {-Project } \\
\text { Team }\end{array}$ \\
\hline $\begin{array}{l}\text { Review and Extension } \\
\text { of Interfaces with other } \\
\text { hardware and software } \\
\text { subsystems }\end{array}$ & -DQAP & $\begin{array}{l}\text {-Meeting } \\
\text {-Woview } \\
\text { sessions }\end{array}$ & $\begin{array}{l}\text {-User } \\
\text {-Project } \\
\text { Team }\end{array}$ \\
\hline
\end{tabular}

[ISA-DQ 5] Review of the Test Plan. In order to test all new data quality characteristics, Test Plan must be reviewed and completed (see Table IX).

TABLE IX. ISA-DQ 5 TASKS

\begin{tabular}{|c|l|l|l|}
\hline Tasks & \multicolumn{1}{|c|}{ Artefact } & \multicolumn{1}{|c|}{$\begin{array}{c}\text { Techniques } \\
\text { and } \\
\text { practices }\end{array}$} & Stakeholder \\
\hline $\begin{array}{l}\text { Review of the } \\
\begin{array}{l}\text { Test Plan for } \\
\text { testing added } \\
\text { characteristics }\end{array}\end{array}$ & -Test Plan review & $\begin{array}{l}\text {-Planning } \\
\text {-Technical } \\
\text { review }\end{array}$ & $\begin{array}{l}\text {-DQAT } \\
\text {-Project } \\
\text { Team }\end{array}$ \\
\hline
\end{tabular}

[ISA-DQ 6] Acceptance of the DQAP. After making all changes for data quality supporting, projects leaders must review them in order to accept them. If so, next process can begin to run (see Table X).

TABLE X. ISA-DQ 6 TASKS

\begin{tabular}{|l|l|l|l|}
\hline Tasks & Artefact & $\begin{array}{c}\text { Techniques } \\
\text { and } \\
\text { practices }\end{array}$ & Stakeholder \\
\hline $\begin{array}{l}\text { Acceptance of } \\
\text { the DQAP }\end{array}$ & -DQAP & -Meeting & $\begin{array}{l}\text {-DQAT } \\
\text {-Project } \\
\text { Manager } \\
\text {-Customer }\end{array}$ \\
\hline
\end{tabular}

\section{Information System Design-Data Quality}

[ISD-DQ] Information System Design-Data Quality. Having finished the activities related to the analysis, 
appropriate reviews must be carried out in design system documents to perform data quality.

[ISD-DQ 1] Review of Physical Data Model. The objective of activities in this process is to review all aspects of physical data model and correct mistakes in designed data components, which have been obtained in the corresponding activities in METRICA V3 (see Table $\mathrm{XI})$.

TABLE XI. ISD-DQ 1 TASKS

\begin{tabular}{|c|c|c|c|}
\hline Tasks & Artefact & $\begin{array}{c}\text { Techniques } \\
\text { and practices }\end{array}$ & Stakeholder \\
\hline $\begin{array}{lr}\text { Review } & \text { of } \\
\text { Physical } & \text { Data } \\
\text { Model } & \end{array}$ & $\begin{array}{l}\text {-DQAP } \\
\text { review }\end{array}$ & $\begin{array}{l}\text {-Working } \\
\text { sessions } \\
\text {-Technical } \\
\text { review }\end{array}$ & $\begin{array}{l}\text {-DQAT } \\
\text {-Project } \\
\text { Team }\end{array}$ \\
\hline $\begin{array}{l}\text { Review and } \\
\text { Extension of Data } \\
\text { Accessibility Paths }\end{array}$ & $\begin{array}{l}\text {-DQAP } \\
\text { review }\end{array}$ & $\begin{array}{l}\text {-Working } \\
\text { sessions } \\
\text {-Technical } \\
\text { review }\end{array}$ & $\begin{array}{l}\text {-DQAT } \\
\text {-Project } \\
\text { Team }\end{array}$ \\
\hline $\begin{array}{ll}\text { Review } & \text { of } \\
\text { Optimization } & \text { of } \\
\text { Data Model } & \end{array}$ & $\begin{array}{l}\text {-DQAP } \\
\text { review }\end{array}$ & $\begin{array}{l}\text {-Working } \\
\text { sessions } \\
\text {-Technical } \\
\text { review }\end{array}$ & $\begin{array}{l}\text {-DQAT } \\
\text {-Project } \\
\text { Team }\end{array}$ \\
\hline $\begin{array}{l}\text { Review of Data } \\
\text { Distribution }\end{array}$ & $\begin{array}{l}\text {-DQAP } \\
\text { review }\end{array}$ & $\begin{array}{l}\text {-Working } \\
\text { sessions } \\
\text {-Technical } \\
\text { review }\end{array}$ & $\begin{array}{l}\text {-DQAT } \\
\text {-Project } \\
\text { Team }\end{array}$ \\
\hline
\end{tabular}

[ISD-DQ 2] Review of Design of Data Managing Processes. This process is similar to the review of data model but in this case it is oriented to managing data processes (see Table XII).

TABLE XII. ISD-DQ 2 TASKS

\begin{tabular}{|l|l|l|l|}
\hline \multicolumn{1}{|c|}{ Tasks } & \multicolumn{1}{|c|}{ Artefact } & \multicolumn{1}{|c|}{$\begin{array}{c}\text { Techniques } \\
\text { and practices }\end{array}$} & Stakeholder \\
\hline $\begin{array}{l}\text { Review of Design } \\
\text { of Data Managing } \\
\text { Processes }\end{array}$ & $\begin{array}{l}\text {-DQAP } \\
\text { review }\end{array}$ & $\begin{array}{l}\text {-Working } \\
\text { sessions } \\
\text {-Technical } \\
\text { review }\end{array}$ & $\begin{array}{l}\text {-DQAT } \\
\text {-Project } \\
\text { Team }\end{array}$ \\
\hline
\end{tabular}

[ISD-DQ 3] Review of Design of Migration Plan and Initial Data Load. The objective of this activity is to review the results of the activity in METRICA V3 in which Design of the Migration Plan and the Initial data Load is done (see Table XIII).

TABLE XIII. ISD-DQ 3 TASKS

\begin{tabular}{|c|c|c|c|}
\hline Tasks & Artefact & $\begin{array}{l}\text { Techniques } \\
\text { and practices }\end{array}$ & Stakeholder \\
\hline $\begin{array}{l}\text { Review of } \\
\text { Detailed Design } \\
\text { of Initial Data } \\
\text { Load }\end{array}$ & $\begin{array}{l}\text {-DQAP } \\
\text { review }\end{array}$ & $\begin{array}{l}\text {-Working } \\
\text { sessions } \\
\text {-Technical } \\
\text { review }\end{array}$ & $\begin{array}{l}\text {-DQAT } \\
\text {-Project Team }\end{array}$ \\
\hline
\end{tabular}

D. Construction of the Data Quality Information System

[ISC-DQ] Construction of the Data Quality Information System. The next step in data quality assurance consists of reviewing the implementation of system components affected by data quality, in order to warrant changes made at analysis and design level are becoming real.
[ISC-DQ 1] Review of Construction of Components and Procedures affected by Data Quality. The objective of this activity is to review the results drawn in corresponding activity of METRICA V3 in order to check the ongoing changes (see Table XIV).

TABLE XIV. ISC-DQ 1 TASKS

\begin{tabular}{|l|l|l|l|}
\hline \multicolumn{1}{|c|}{ Tasks } & \multicolumn{1}{|c|}{ Artefact } & $\begin{array}{l}\text { Techniques } \\
\text { and practices }\end{array}$ & Stakeholder \\
\hline $\begin{array}{l}\text { Review of } \\
\text { construction of } \\
\text { components } \\
\text { affected by DQ. }\end{array}$ & $\begin{array}{l}\text {-Components } \\
\text { review }\end{array}$ & $\begin{array}{l}\text {-Working } \\
\text { sessions }\end{array}$ & $\begin{array}{l}\text {-Project } \\
\text { Team }\end{array}$ \\
\hline
\end{tabular}

[ISC-DQ 2] Review of Testing for Validating data quality aspects. The aim is to guarantee that testing include tests for validating data quality (see Table XV).

TABLE XV. ISC-DQ 2 TASKS

\begin{tabular}{|c|c|c|c|}
\hline Tasks & Artefact & $\begin{array}{c}\text { Techniques } \\
\text { and practices }\end{array}$ & Stakeholder \\
\hline $\begin{array}{l}\text { Review of } \\
\text { testing for } \\
\text { validating data } \\
\text { quality aspects. }\end{array}$ & -Testing review & $\begin{array}{l}\text {-Working } \\
\text { sessions }\end{array}$ & $\begin{array}{l}\text {-DQAT } \\
\text {-Project } \\
\text { Team }\end{array}$ \\
\hline
\end{tabular}

[ISC-DQ 3] Review of User Manuals. In order to achieve Total Data Quality Management, it is necessary to create a data quality environment. First step to get this objective is to try users become aware of data quality is essential in their work, like a work philosophy. The user manual, the principal information source for user learning about system exploitation, goes towards achieving this goal. The objective of this activity is to review the user manual in order to emphasize the aspects related to DQ to create a data quality environment (see Table XVI).

TABLE XVI. ISC-DQ 3 TASKS

\begin{tabular}{|c|l|l|l|}
\hline Tasks & \multicolumn{1}{|c|}{ Artefact } & \multicolumn{1}{|c|}{$\begin{array}{c}\text { Techniques } \\
\text { and practices }\end{array}$} & Stakeholder \\
\hline $\begin{array}{l}\text { Review of } \\
\text { User Manuals } \\
\text { affected by }\end{array}$ & -User Manuals & $\begin{array}{l}\text {-Working } \\
\text { sessions } \\
\text { Data Quality. }\end{array}$ & -Project Team \\
\hline
\end{tabular}

[ISC-DQ 4] Review of the Training for Final Users. Another source of information for users may be courses in which the functioning of the system is explained to them. Final users are trained in the typical procedures associated with exploitation of the system. This provides a good moment to promote a data quality philosophy which will favour the correct exploitation of the system. The objective of this activity is to review the training scheme in order to add the necessary data quality connotations so that users make aware of data quality (see Table XVII).

TABLE XVII. ISC-DQ 4 TASKS

\begin{tabular}{|lr|l|l|l|}
\hline \multicolumn{2}{|c|}{ Tasks } & \multicolumn{1}{|c|}{ Artefact } & \multicolumn{1}{|c|}{$\begin{array}{c}\text { Techniques } \\
\text { and practices }\end{array}$} & \multicolumn{1}{c|}{ Stakeholder } \\
\hline $\begin{array}{l}\text { Review of } \\
\text { Training for }\end{array}$ & -Training & -Working & -User \\
final users. & & sessions & -DQAT \\
-Meeting & -Project Team \\
\hline
\end{tabular}


[ISC-DQ 5] Presentation and Acceptation of Data Quality Modifications. The objective of this activity is to explain the impact of the DQAP on the Information System Plan to the technical and administrative personnel of the project, for its acceptation and correction (see Table XVIII).

TABLE XVIII. ISC-DQ 5 TASKS

\begin{tabular}{|l|l|l|l|}
\hline \multicolumn{1}{|c|}{ Tasks } & \multicolumn{1}{|c|}{ Artefact } & \multicolumn{1}{c|}{$\begin{array}{c}\text { Techniques } \\
\text { and practices }\end{array}$} & Stakeholder \\
\hline $\begin{array}{l}\text { Approval of } \\
\text { Data Quality } \\
\text { Modifications }\end{array}$ & -Approval & -Presentation & $\begin{array}{l}\text {-Customer } \\
\text {-Project } \\
\text { Manager } \\
\text {-DQAT. }\end{array}$ \\
\hline
\end{tabular}

E. Maintenance of the Data Quality Information System [ISM-DQ] Maintenance of the Data Quality Information System. This process must be considered in the corresponding maintenance process of METRICA V3, in order to examine maintenance request from data quality view point to analyse if non data quality is the origin of the problem.

[ISM-DQ 1] Registration and Assignment of Maintenance Request. When faults are detected, final user must present a maintenance request in order to resolve the problem. This request may be directed to the project team or directly to the Data Quality Assurance Team if fault is suspicious to be due to a poor data quality situation (see Table XIX).

TABLE XIX. ISM-DQ 1 TASKS

\begin{tabular}{|c|c|c|c|}
\hline Tasks & Artefact & $\begin{array}{c}\text { Techniques and } \\
\text { practices }\end{array}$ & Stakeholder \\
\hline $\begin{array}{lr}\text { Extension } & \text { of } \\
\text { Maintenance Request } \\
\text { affected by Dara } \\
\text { Quality }\end{array}$ & $\begin{array}{l}\text {-Maintenance } \\
\text { Plan review }\end{array}$ & $\begin{array}{l}\text {-Working } \\
\text { sessions }\end{array}$ & $\begin{array}{l}\text {-DQAT } \\
\text {-Project } \\
\text { Team }\end{array}$ \\
\hline
\end{tabular}

[ISM-DQ 2] Request Analysis and Proposed Solution Analysis. If maintenance request is finally directed to the DQAT, then this group must study it in order to locate the problem and provide a possible solution (see Table XX).

TABLE XX. ISM-DQ 2 TASKS

\begin{tabular}{|c|c|c|c|}
\hline Tasks & Artefact & $\begin{array}{c}\text { Techniques } \\
\text { and practices }\end{array}$ & Stakeholder \\
\hline $\begin{array}{l}\text { Extension of } \\
\text { Maintenance } \\
\text { Solution Analysis } \\
\text { affected by DQ }\end{array}$ & $\begin{array}{l}\text {-Maintenance } \\
\text { Plan review }\end{array}$ & $\begin{array}{l}\text {-Working } \\
\text { sessions } \\
\text {-Technical } \\
\text { analysis }\end{array}$ & $\begin{array}{l}\text {-DQAT } \\
\text {-Project } \\
\text { Team }\end{array}$ \\
\hline
\end{tabular}
proposed solution is right, the appropriate modifications to the development of the system must be made in order for it to be effective (see Table XXI).

TABLE XXI. ISM-DQ 3 TASKS

\begin{tabular}{|c|c|c|c|}
\hline Tasks & Artefact & $\begin{array}{l}\text { Techniques and } \\
\text { practices }\end{array}$ & Stakeholder \\
\hline $\begin{array}{lr}\text { Extension } & \text { of } \\
\text { Maintenance } & \\
\text { Solution } & \text { Plan } \\
\text { affected by DQ } & \\
\end{array}$ & $\begin{array}{l}\text { Maintenance } \\
\text { Plan review }\end{array}$ & $\begin{array}{l}\text {-Working sessions } \\
\text {-Technical } \\
\text { analysis }\end{array}$ & $\begin{array}{l}\text {-DQAT } \\
\text {-Project } \\
\text { Team }\end{array}$ \\
\hline
\end{tabular}

This Data Quality Interface is optional. So, a development team might use it or no; but if they do, they are recommended to use it following the proposed order.

\section{Discussion AND CONCLUSION}

At present, Information Systems in organization are as important as proper organization. Data and information are fundamental assets of any organization, and is the raw material of these information systems. Therefore, the data must have enough quality to achieve quality information systems that satisfy the information needs of users.

To address this problem, this paper has proposed a framework for incorporating data quality assurance aspects (through user requirements), in the development stages of an information system. It has built an interface for software development methodology.

An interface (a set of process and activities with a defined purpose) for a well defined and proved and widely used methodology has been presented. The goal of this interface is to assurance data quality for a developed system.

The proposed data quality interface is specified at level tasks, products and roles; therefore, it is possible to extend the interface to other proposed software development methodologies similar to METRICA V3.

\section{REFERENCES}

[1] Lee, Y.W., et al., Journey to Data Quality. 2006, Cambridge, MA, USA: Massachussets Institute of Technology.

[2] Kahn, B.K., D.M. Strong, and R.Y. Wang, Information Quality Benchmarks: Product and Service Performance. Communications of the ACM, 2002. 45(4ve): p. 184-192.

[3] Pipino, L., Y. Lee, and R. Wang, Data Quality Assessment. Communications of the ACM, 2002. 45(4): p. 211-218.

[4] Ballou, D.P. and H.L. Pazer, Modeling Completeness versus Consistency Tradeoffs in Information Decision Contexts IEEE Transactions on Knowledge and Data Engineering 200315 (1): p. 240-243

[5] Shankaranayanan, G. and Y. Cai. A Web Services Application for the Data Quality Management in the B2B Networked Environment. in 38th Hawaii International Conference on System Sciences (HICSS-38 2005). 2005. Big Island, HI, USA: IEEE Computer Society.

[6] Scannapieco, M. and L. Berti-Équille, Report from the First and Second International Workshops on Information Quality in Information Systems- IQIS 2004 and IQIS 2005 in Conjunction with ACM SIGMOD/PODS Conferences. SIGMOD RECORD, 2006. 35(2): p. 50-52.

[7] Cai, Y. and G. Shankaranarayanan, Managing data quality in inter-organisational data networks. International Journal of Information Quality, 2007. 1(3): p. 254 - 271.

[8] Burgess, M.S.E., W.A. Gray, and N.J. Fiddian. A flexible quality framework for use within information retrieval. in 
Eighth International Conference on Information Quality (ICIQ'03). 2003. MIT, Cambridge, MA, USA.

[9] Caballero, I., Ó. Gómez, and M. Piattini. Getting Better Information Quality by Assessing and Improving Information Quality Management. in Ninth International Conference on Information Quality (ICIQ'04). 2004. MIT, Cambridge, MA, USA.

[10] Strong, D., Y. Lee, and R. Wang, Ten Potholes in the Road to Information Quality. IEEE Computer, 1997: p. 38-46.

[11] Eppler, M. and M. Helfert. A Classification and Analysis of Data Quality Costs. in International Conference on Information Quality. 2004. MIT, Cambridge, MA, USA.

[12] MAP. METRICA. VERSION 3. Metodología de Planificación, Desarrollo y Mantenimiento de Sistemas de Información. 2000 [cited; Available from: http://www.csi.map.es/csi/metrica3/.

[13] Rochfeld, A. and H. Tardieu, Merise: An information system design and development methodology., in Information Manag. 1983. p. 143-159.
[14] Goodland, M. and C. Slater, SSADM Version 4: A Practical Approach. 1995, London: McGraw-Hill.

[15] ISO/IEC, ISO/IEC 12207. International Standard. Software Life Cycle Processes. 1995, International Standard Organziation/International Electrotechnical Committee: Geneve.

[16] ISO/IEC, (1998):15504-2: Information TechnologySoftware Process Assessment-Part 2: A Reference Model for Processes and Process Capability. 1998.

[17] 17 ISO/IEC. ISO/IEC Quality Management Systems Fundamentals and Vocabulary. ISO 9000:2000. 2000 [cited; Available from: http://www.iso.org.

[18] ISO/IEC. ISO/IEC Quality Management Systems Requirements. ISO 9001:2000. $2000 \quad$ [cited; Available from: http://www.iso.org.

[19] Boar, B.H., The art of strategic planning for information technology: crafting strategy for the 90s. 1993: John Wiley and Sons, Inc. 